\title{
Avaliação de uma segunda lavagem uterina sobre a recuperacão de embriões em vacas Nelore
}

\author{
Evaluation of a second uterine flushing on embryo recovery in \\ Nelore cows
}

\author{
Antônio Germano Wünsche Júnior ${ }^{1}$; Thales Ricardo Rigo Barreiros ${ }^{2 *}$; \\ Gustavo de Arruda Bezerra ${ }^{3}$; Jefferson Roela Azevedo ${ }^{3}$; \\ Ana Carolina Santos Oliveira ${ }^{4}$; Wanessa Blaschi ${ }^{5}$; Marcelo Marcondes Seneda ${ }^{6}$
}

\section{Resumo}

O objetivo do presente experimento foi verificar a viabilidade de uma segunda lavagem uterina sobre a recuperação de embriões. Doadoras cíclicas da raça Nelore $(n=6)$ foram mantidas a pasto no município de Mamborê, Estado do Paraná. Os animais foram submetidos a 2 tratamentos de superovulação com intervalo de 80 dias. Em um dia aleatório do ciclo estral (Dia 0) as doadoras receberam um dispositivo intravaginal contendo 1,9 g de progesterona (CIDR; Pfizer, Brasil). Vinte e quatro horas após (Dia 1) administrou-se 2,5 mg de benzoato de Estradiol (Estrogin; Farmavet, Brasil) por via intramuscular (IM). Entre Dia 5 e Dia 9 realizou-se a superovulação com 250 UI de FSH IM em doses decrescentes (Pluset; Calier, Brasil). Administrou-se 25 mg de dinoprost IM (Lutalyse; Pfizer, Brasil) na manhã do D7 e 12 hs depois, simultaneamente à retirada do dispositivo de progesterona. As vacas foram inseminadas 12 e 24 horas após o início das manifestações estrais. A primeira lavagem uterina foi realizada no sétimo dia após a inseminação artificial, com o posicionamento das sondas de Foley caudal à bifurcação do útero. Em cada lavagem, foram usados $1.000 \mathrm{ml}$ de DPBS (Embriocare; Cultilab, Brasil) em sistema fechado conectado a um filtro coletor de $70 \mu \mathrm{m}$. O lavado uterino foi avaliado com auxílio de um estereomicroscópio binocular (SMZ 654, Nikon, Japão) e os embriões foram classificados seguindo os padrões da IETS (1998). Vinte e quatro horas após, a segunda lavagem uterina foi realizada seguindo o mesmo procedimento. Os resultados foram analisados pelo teste $t$-Student com significância de $5 \%$. Após 12 superovulações, foram obtidas 131 estruturas sendo 91,4\% (120/131) na primeira lavagem e $8,4 \%$ (11/131) no segundo procedimento. A segunda lavagem uterina permitiu a recuperação de um número reduzido de embriões, condicionando o procedimento a embriões cujo valor justifique o acréscimo de trabalho.

Palavras-chave: Bovino, embriões, colheita

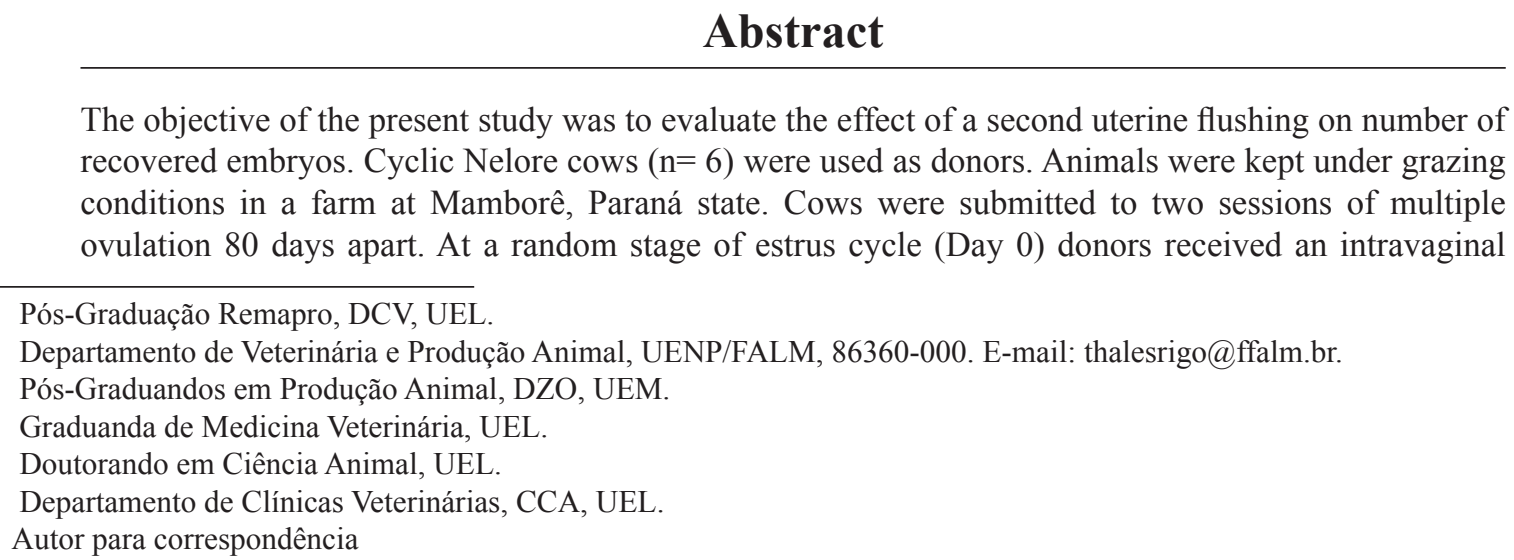

Abstract

The objective of the present study was to evaluate the effect of a second uterine flushing on number of recovered embryos. Cyclic Nelore cows $(\mathrm{n}=6)$ were used as donors. Animals were kept under grazing conditions in a farm at Mamborê, Paraná state. Cows were submitted to two sessions of multiple ovulation 80 days apart. At a random stage of estrus cycle (Day 0) donors received an intravaginal 
device with $1.9 \mathrm{~g}$ of progesterone (CIDR; Pfizer; Brasil). Twenty four hours later (Day 1) cows were injected with $2.5 \mathrm{mg} / \mathrm{IM}$ of Estradiol Benzoate (Estrogin, Farmavet, Brasil). Between Day 5 and Day 9, donors received decreasing doses of 250 UI of FSH (Pluset; Calier, Brasil). On Days 7 and cows received $25 \mathrm{mg} / \mathrm{IM}$ of dinoprost (Lutalyse, Pfizer, Brasil) and progesterone implants were removed 12 hs later. Artificial insemination was performed 12 and 24 hours after estrus detection. The first embryo recovery was performed 7 days after AI with the Foley catheter positioned in the uterine body. A total of $1.000 \mathrm{~mL}$ of PBS (Embriocare, Cultilab, Brasil) was used in a closed system of uterine flushing, with a $70 \mu \mathrm{m}$ filter. Recovered uterine fluid was evaluated under a stereomicroscope (SMZ 654, Nikon, Japan) and embryos were classified accord to IETS (1998). Twenty four hours later, the second uterine flushing was performed following the same procedure. Results were analyzed by $t$-Student test $(\mathrm{p}<0,05)$. After 12 superovulation procedures, we obtained a total of 131 ova/embryo, being 91.4\% (120/131) from the first uterine flushing and $8.4 \%$ (11/131) from the second one. We conclude that the second uterine flushing provided a small improvement on the number of recovered embryos. The feasibility of this method is restricted to special conditions, when the value of the embryos supports the additional work.

Key words: Cattle, embryo, uterine flushing

\section{Introdução}

O Brasil tem-se destacado na produção de embriões bovinos, sobretudo pelos resultados promissores obtidos com produção in vitro na raça Nelore. Entretanto, a produção in vivo de embriões é amplamente utilizada, por apresentar menor custo de produção (BOUSQUET et al., 1999; NONATO JÚNIOR, 2005). Avanços na compreensão da fisiologia reprodutiva das fềmeas zebuínas contribuíram para esta situação de destaque observada no cenário nacional, pois viabilizaram a elaboração de novos protocolos de superovulação, permitindo melhor controle farmacológico do crescimento folicular e do ciclo estral (PINHEIRO et al., 1998; BURATINI et al., 2000; BARUSELLI et al., 2006).

Apesar desses avanços, a produção in vivo de embriões poderia ser incrementada. A redução das variações nas respostas superovulatórias e uma maior eficiência na recuperação de embriões constituem os principais desafios da técnica (MAPLETOFT; STEWARD; ADAMS, 2002; REICHENBACH, 2003; CASTRO NETO et al., 2005).

O método não cirúrgico de lavagem uterina permitiu um considerável avanço na recuperação embrionária(GREVE;LEHN-JENSEN;RASBECH, 1977). Porém, após mais de duas décadas, poucas modificações foram sugeridas para incrementar o procedimento, verificando-se variações de 20 a $80 \%$ nas taxas de recuperação de embriões por folículo ovulado (SARTORI et al.,2003). Para aumentar a eficiência da recuperação dos embriões, algumas modificações na lavagem uterina têm sido propostas, como posicionamento do catéter no corpo ou no corno do útero e uma dupla lavagem uterina, com a segunda 30 minutos após a primeira (SARTORI et al., 2003; CASTRO NETO et al., 2005). Uma das hipóteses desses autores seria a recuperação de estruturas na junção útero-tubárica, referindo-se aos embriões em trânsito da tuba uterina para o útero, quando mais de uma lavagem é realizada.

A hipótese deste trabalho é a possibilidade de recuperação de embriões eventualmente presentes na tuba uterina ao sétimo dia pós IA. Nosso objetivo foi investigar a eficiência de uma segunda lavagem uterina, realizada 24 horas após a primeira, quanto ao número de embriões obtidos.

\section{Material e Métodos}

Local

$\mathrm{O}$ presente experimento foi realizado no município de Mamborê (latitude 24¹9'9'S; longitude $52^{\circ} 31^{\prime} 48^{\prime}$ 'W, altitude 762m, BIM IBGE, 2000), Estado do Paraná, durante o período de dezembro 2005 a fevereiro 2006.

\section{Animais}

Foram utilizadas seis vacas da raça Nelore $(B o s$ 
taurus indicus) sem utilização prévia na colheita de embriões, com idade média de 80 meses (40 a 120) e escore da condição corporal entre 3 e 4 (escala de 1 a 5); (LOWMAN; SCOTT; SOMERVILLE, 1976). Estas vacas eram mantidas em pastagem de Cynodon nlemfuensis e recebiam suplementação mineral à vontade contendo $9 \%$ de fósforo. Estes animais foram submetidos a exames de palpação e ultrasonografia transretal (Aloka SSD-500, $5 \mathrm{MHz}$ ) para detecção de possíveis anormalidades no trato genital feminino, de forma que todos se apresentaram com o aparelho reprodutor morfologicamente normal. A regularidade dos ciclos estrais foi confirmada pela observação visual de pelo menos três comportamentos estrais em intervalos de 18 a 23 dias.

\section{Protocolo de superovulação}

As seis vacas utilizadas neste experimento foram submetidas a duas sessões de superovulação, com intervalo de 80 dias, totalizando 12 procedimentos. Estes animais receberam pela manhã um dispositivo intravaginal de 1,9 g de progesterona (CIDR; Pfizer, Brasil). Vinte e quatro horas após, administrou-se 2,5 mg de benzoato de estradiol (Estrogin; Farmavet, Brasil) por via intramuscular (IM). Cinco dias depois do início do tratamento, os animais receberam 250 UI de extrato hipofisário suíno - FSH - (Pluset; Calier, Espanha) IM, em oito doses decrescentes, a cada 12 horas. Quarenta e oito e 60 horas depois do início da aplicação do FSH, os animais receberam $25 \mathrm{mg}$ de dinoprost IM (Lutalyse; Pfizer, Brasil). Os dispositivos de progesterona foram retirados 12 horas depois da primeira aplicação de dinaprost. As vacas foram mantidas em observação de cio nas 72 horas seguintes e inseminadas 12 e 24 horas após o início das manifestações estrais. O protocolo de superovulação está representado na Figura 1.
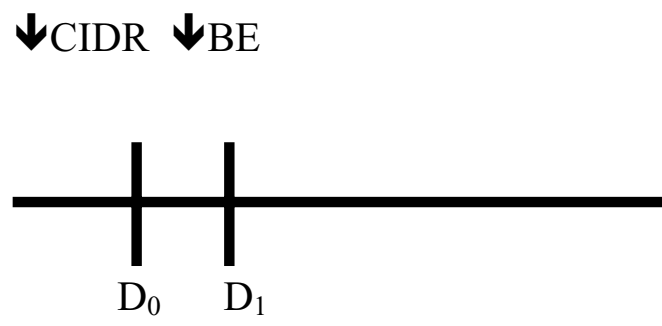

* m - manha, $\mathrm{t}$ - tarde
$\operatorname{PGF}(\mathrm{m} / \mathrm{t})^{*}$ $\uparrow \operatorname{CIDR}(\mathrm{t})$

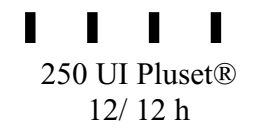

$\begin{array}{llllll}\mathrm{D}_{5} & \mathrm{D}_{6} & \mathrm{D}_{7} & \mathrm{D}_{8} & \mathrm{D}_{9} & \mathrm{D}_{10}\end{array}$

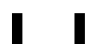

colheitas de embriões

IA 12 e 24 h após início do estro

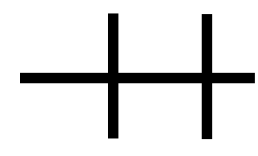

$\mathrm{D}_{16} \quad \mathrm{D}_{17}$

Figura 1. Representação esquemática do protocolo de superovulação utilizado em vacas da raça Nelore, Mamborê/ PR.

\section{Obtenção dos embriões}

Todas as doadoras foram submetidas ao mesmo procedimento, conduzido pelo mesmo profissional, aproximadamente sete dias após a inseminação artificial (D7). Inicialmente avaliou-se a resposta à terapia superovulatória por palpação retal e em seguida foi realizada a anestesia epidural baixa empregando $4 \mathrm{~mL}$ de lidocaína 2\% (Anestésico L;
Eurofarma, Brasil). A limpeza da vulva e do períneo foi procedida com o uso de sabão neutro e água para remoção das sujidades e posterior anti-sepsia com álcool $70^{\circ}$. Foi adotada a colheita de embriões por via transcervical com sistema fechado, utilizandose sondas de látex com duas vias (Sonda de Foley, Rüsch, Alemanha) posicionadas no corpo do útero, conectadas em equipos específicos (Equipo em Y, Cultilab, Brasil) e copo coletor com filtros de $70 \mu \mathrm{m}$ 
(Coletor de embriões, Cultilab, Brasil). Em cada colheita, foram usados $1.000 \mathrm{~mL}$ de meio DPBS (tampão fosfato salino, glicose, piruvato de sódio, penicilina $\mathrm{G}$ potássica, gentamicina e anfotericina B); (Embriocare, Cultilab, Brasil), previamente aquecidos a $30^{\circ} \mathrm{C}$. Logo após a colheita, os filtros foram lavados com aproximadamente $60 \mathrm{~mL}$ de DPBS e a fração do lavado uterino foi transferida para placas de Petri estéreis de 100 x 20 mm (Corning, EUA). A procura dos embriões foi feita com auxílio de um esteromicroscópio binocular no aumento de 15 vezes (SMZ 654, Nikon, Japão). As estruturas obtidas foram transferiras para placas de Petri de 35 x $10 \mathrm{~mm}$ (Corning, EUA) e mantidas em solução de manutenção contendo DPBS e $0,4 \%$ de albumina bovina (Holding Plus, AB Technology; Brasil) para posterior classificação em aumento de 80 vezes, segundo os padrões da Sociedade Internacional de
Transferência de Embriões (IETS, 1998). Todos os procedimentos descritos foram repetidos vinte e quatro horas depois da primeira lavagem (D8).

\section{Análise estatística}

A análise estatística foi realizada utilizando-se o software Sigma Stat for windows 2.0. As médias das estruturas totais, viáveis e inviáveis recuperadas na primeira e nas duas colheitas foram analisadas pelos testes $t$-Student com significância de 5\%.

\section{Resultados e Discussão}

Foram realizados 24 procedimentos de lavagem uterina, sendo 12 no sétimo e 12 no oitavo dia pós IA. Cento e trinta e uma estruturas foram recuperadas, sendo 91,6\% (120/131) no primeiro (D7) e 8,4\% (11/131) no segundo procedimento (D8); (Figura 2).

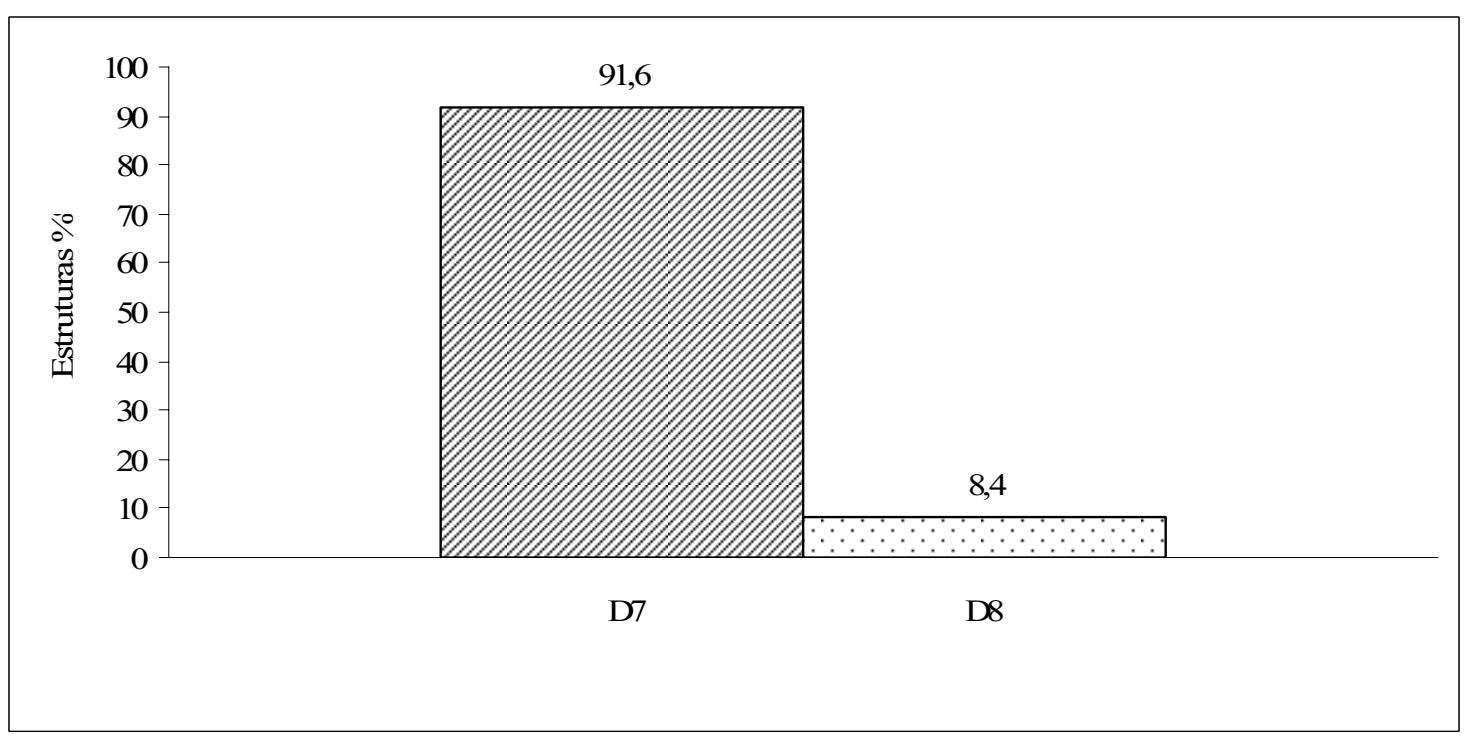

Figura 2. Proporções de estruturas recuperadas na primeira e segunda colheita de embriões em vacas da raça Nelore, dezembro de 2005 a fevereiro de 2006, Mamborê/PR.

Desde a introdução da colheita não cirúrgica ou transcervical de embriões, pequenas modificações na técnica foram descritas durante as três últimas décadas. Embora o chamado "sistema fechado" de lavagem uterina seja uma técnica relativamente simples, um problema desafiador tem sido constatado por vários pesquisadores. Trata-se da variação na taxa de recuperação embrionária, ou seja, a não correspondência entre o número de folículos ovulados e as estruturas recuperadas no 
lavado uterino no sétimo dia após inseminação. As variações relatadas são da ordem de 20 a $80 \%$ (SARTORI et al., 2003).

Uma possível explicação para tal seria o local de posicionamento da sonda de Foley no momento da lavagem do útero. Hay et al. (1990) compararam a recuperação de estruturas com a sonda de Foley posicionada no corpo ou no corno do útero e não encontraram diferenças significativas quanto aos embriões recuperados. No entanto, Sartori et al. (2003) obtiveram maior eficiência com posicionamento do catéter na base do corno uterino, sendo o posicionamento próximo à tuba uterina menos eficiente. A comparação direta dos métodos de posicionamento do catéter é bastante crítica, pois envolve inclusão de vários outros aspectos, como a preferência do profissional, a faixa etária dos animais e o tipo do catéter utilizado. Em nosso trabalho, o posicionamento do catéter no corpo uterino mostrou-se adequado, pois obtivemos uma média de embriões compatível com resultados de outros autores (VISINTIN et al., 1999).
Nosso principal objetivo consistiu em avaliar a presença de embriões com uma segunda lavagem uterina. Por este motivo, a taxa de recuperação (número de estruturas por folículo ovulado) não foi analisada. Apesar disto, destacamos o nível satisfatório de eficiência na primeira lavagem, pois a média de estruturas obtidas $(10 \pm 0,9)$ foi superior às citadas por Castro Neto et al. (2005). Por este motivo, acreditamos que todos os embriões presentes no útero à primeira lavagem foram realmente recuperados ao sétimo dia pós IA.

A segunda lavagem não aumentou significativamente as médias de estruturas recuperadas. Considerando-se, respectivamente, primeira lavagem e total das duas lavagens, foram obtidas $10 \pm 0,9$ vs $10,9 \pm 1,1$ para estruturas totais e $6,3 \pm 1,0$ vs. $6,75 \pm 1,4$ para embriões viáveis $(\mathrm{P}>0,05)$. Da mesma forma, não houve diferença na média de embriões degenerados e oócitos não fecundados. Estes dados estão na descritos na Tabela 1.

Tabela 1. Médias e erro padrão das estruturas totais, embriões viáveis, embriões degenerados e ovócitos não fertilizados obtidos nas colheitas de embriões em D7 e D8 em vacas da raça Nelore, dezembro de 2005 a fevereiro de 2006, Mamborê/PR.

\begin{tabular}{|c|c|c|}
\hline \multirow{2}{*}{ Parâmetro } & \multicolumn{2}{|c|}{ Colheita de embriões } \\
\hline & D7 & $\mathrm{D} 7+\mathrm{D} 8$ \\
\hline $\mathrm{N}$ & 12 & 12 \\
\hline Estruturas totais & $10 \pm 0,9$ & $10,9 \pm 1,1$ \\
\hline Embriões viáveis & $6,25 \pm 1,0$ & $7,0 \pm 1,4$ \\
\hline $\begin{array}{l}\text { Embriões degenerados e ovócitos } \\
\text { não fecundados }\end{array}$ & $3,7 \pm 0,9$ & $3,9 \pm 0,9$ \\
\hline
\end{tabular}

$(\mathrm{P}>0,05)$

Estes resultados foram inferiores aos reportados por outros pesquisadores, com experimentos referentes à modificações na ténica de lavagem uterina. Um aumento de $30 \%$ de embriões recuperados foi obtido por Subramaniam, Devarajan e Mohanan (1991), com uma segunda lavagem em D8. No entanto, esses autores divulgaram seus dados apenas sob forma de resumo, inviabilizando uma comparação mais detalhada. Castro Neto et al. (2005) recuperaram um número significativamente maior de embriões na segunda lavagem, sendo esta realizada apenas 30 minutos da primeira, mas com o diferencial do útero permanecer repleto de PBS neste intervalo de tempo. Pelas diferenças de método, não é possível uma comparação direta com nossos resultados. 
A retenção de embriões na tuba uterina tem sido alvo de estudo de alguns pesquisadores. Esta possível retenção resultaria de uma migração tardia dos embriões para o útero, pois as altas concentrações de estradiol, após a superovulação, poderiam alterar a migração e a viabilidade dos embriões em doadoras submetidas à superovulação (HACKETT et al., 1993). Adicionalmente, a migração dos embriões poderia estar associada com a viabilidade e número dos mesmos (MACMILLAN; PETERSON, 1999). A possibilidade de embriões retidos nas tubas uterinas foi testada por Hackett et al. (1993) ao lavarem as tubas uterinas, a extremidade do corno uterino e o restante do corno uterino quatro, cinco e seis dias após a inseminação artificial de vacas Holandesas. No quarto dia, $60 \%$ dos embriões foram encontrados nas tubas uterinas. No quinto dia, $80 \%$ dos embriões estavam no útero, e no sexto dia, este número subiu para 91\%. Alvarez, Carvalho e Carvalho (1999) também verificaram estes aspectos e encontraram $4,2 \%$ das estruturas recuperadas localizadas nas tubas uterinas em $25 \%$ de vacas eutanasiadas no dia da colheita. Comparando estes dados, consideramos ser possível inferir que os embriões recuperados na segunda lavagem estivessem realmente na tuba uterina ao sétimo após a IA.

Um dos aspectos relevantes deste experimento foi o caráter essencialmente acadêmico do mesmo. Desde a seleção dos animais até a condução dos procedimentos de lavagem uterina, todas as etapas foram realizadas com o intuito de se testar a hipótese. Consideramos, desta forma, a perspectiva promissora de repetição do mesmo em uma central comercial de embriões. Embora as restrições naturais quanto ao rigor experimental, esta alternativa poderia contribuir com a ampliação do número de procedimentos. Além disso, em procedimentos de larga escala, variações numéricas não significativas estatisticamente podem representar substancial acréscimo. Assim, o incremento de $8 \%$ em número de embriões, obtido com a segunda lavagem, poderia justificar o acréscimo de trabalho, dependendo do valor dos embriões em questão.
A hipótese de recuperação de embriões com uma segunda lavagem 24 horas após a primeira se confirmou. No entanto, o reduzido acréscimo verificado limita o procedimento para embriões de elevado valor.

\section{Agradecimentos}

Laboratórios Pfizer, Divisão de Saúde Animal.

\section{Referências}

AlvarEZ, R. H.; CARVALHO, J. B. P.; CARVALHO, M. I. A. B. Embriões retidos no oviduto como fator de variação na taxa final de recuperação após lavagem uterina de vacas superovuladas. Boletim da Indústria Animal, Nova Odessa, v. 56, n. 1, p. 89-93, 1999.

BARUSELli, P. S.; SÁ FILHO, M.; MARTINS, C.; NASSER, L.; NOGUEIRA, M. G. F.; BARROS, C. M.; BO, G. A. Superovulation and embryo transfer in Bos indicus cattle. Theriogenology, Stoneham, v. 65, n. 1, p. 77-88, 2006.

BIM IBGE 2000. Disponível em: <http://www.mambore. pr.probrasil.com.br/>. Acesso em: $21 \mathrm{dez} .2005$.

BOUSQUET, D.; TWAGIRAMUNGU, H.; MORIN, N.; BRISSON, C.; CARBONEAU, G.; DUROCHER, J. In vitro embryo production in the cow: an effective alternative to the conventional embryo production approach. Theriogenology, Stoneham, v. 51, n. 1, p. 5970, 1999.

BURATINI JÚNIOR, J.; PRICE, C. A.; VISINTIN, J. A.; BÓ, G. A. Effects of dominant follicle aspiration and treatment with recombinant bovine somatotropin (BST) on ovarian follicular development in Nelore (Bos indicus) heifers. Theriogenology, Stoneham, v. 54, n. 3, p. 421-431, 2000.

CASTRO NETO, A. S.; SANCHES, B. V.; BINELLI, M.; SENEDA, M. M.; PERRI, S. H.; GARCIA, J. F. Improvement in embryo recovery using double uterine flushing. Theriogenology, Stoneham, v. 63, n. 5, p. 1249$1255,2005$.

GREVE, T.; LEHN-JENSEN, H.; RASBECH, N. O. Nonsurgical recovery of bovine embryos. Theriogenology, Stoneham, v. 7, n. 4, p. 239-250, 1977.

HACKETT, A. J.; DURNFORD, R.; MAPLETOFT, R. J.; MARCUS, G. J. Location and status of embryos in the genital tract of superovulated cows 4 to 6 days after insemination. Theriogenology, Stoneham, v. 40, n. 6, p. 1147-1153, 1993. 
HAY, J. H.; PHELPS, D. A.; HANKS, D. R.; FOOTE, W. D. Sequential uterine horn versus simultaneous total uterine flush to recover bovine embryos nonsurgically. Theriogenology, Stoneham, v. 33, n. 2, p. 563-567, 1990.

LOWMAN, B. G.; SCOTT, N. A.; SOMERVILLE, S. H. Condition scoring of cattle. Bulletin East of Scotland College of Agriculture, Edinburgh, v. 6, n. 1, p. 1-31, 1976.

MACMILLAN, W. H.; PETERSON, A. J. Transuterine embryo migration in recipient cattle. Theriogenology, Stoneham, v. 51, n. 8, p. 1577-1586, 1999.

MAPLETOFT, R. J.; STEWARD, K. B.; ADAMS, G. P. Recent advances in the superovulation in cattle. Reproduction Nutrition and Development, Paris, v. 42, n. 3, p. 601-611, 2002.

NONATO JÚNIOR, I. Produção de embriões em vacas Nelore (Bos taurus indicus) com a associação dos métodos in vivo e in vitro. 2005. Dissertação (Mestrado em Pós-graduação em Ciência Animal) - Universidade Estadual de Londrina, Londrina.

PINHEIRO, O. L.; BARROS, C. M.; FIGUEIREDO, R. A.; VALLE, E. R.; ENCARNAÇÃO, R. O.; PADOVANI, C. R. Estrus behavior and the to ovulation interval in Nelore cattle (Bos indicus) with natural estrus or estrus induced with prostaglandin F2 $\alpha$ or norgestomet and estradiol valerate. Theriogenology, Stoneham, v. 49, n. 3, p. 667-681, 1998.
REICHENBACH, H. D. Transferência e congelamento de embriões bovinos: considerações práticas. Acta Scientiae Veterinariae, Porto Alegre, v. 31, n. 1, p. 15-21, 2003.

SARTORI, R.; SUÁREZ-FERNÁNDEZ, C. A.; MONSON, R. L.; GUENTHER, J. N.; ROSA, G. J. M.; WILTBANK, M. C. Improvement in recovery of embryos/ ova using a shallow uterine horn flushing technique in superovulated Holstein heifers. Theriogenology, Stoneham, v. 60, n. 7, p. 1319-1330, 2003.

SUBRAMANIAM, A.; DEVARAJAN, K. P.; MOHANAN, M. Repeat flushing and embryo recovery (RFER) in cross-bred donors cows (Bos indicus x Bos taurus). Theriogenology, Stoneham, v. 35, n. 1, p. 277, 1991.

VISINTIN, J. A.; ARRUDA, R. P.; MADUREIRA, E. H.; MIZUTA, K.; CELEGHINI, E. C. C.; ASSUMPÇÃO, M. E. O. D.; GUSMÕES, P. P. G.; CANDINI, P. H. Superovulation of Nelore heifers with different FSH/ LH doses and embryo freezing by one-step method with ethylene-glycol. Brazilian Journal of Veterinary Research and Animal Science, São Paulo, v. 36, n. 5, 1999. 
